\title{
Germanica
}

\section{Bovarysme et snobisme, deux symptômes de la nervosité des nations. Étude de cas : Der Mann ohne Eigenschaften}

Till R. Kuhnle

\section{(2) OpenEdition}

Journals

Édition électronique

URL : http://journals.openedition.org/germanica/1370

DOI : $10.4000 /$ germanica. 1370

ISSN : 2107-0784

Éditeur

Université de Lille

Édition imprimée

Date de publication : 31 décembre 2011

Pagination : 55-68

ISBN : 9782913857285

ISSN : 0984-2632

Référence électronique

Till R. Kuhnle, "Bovarysme et snobisme, deux symptômes de la nervosité des nations. Étude de cas Der Mann ohne Eigenschaften», Germanica [En ligne], 49 | 2011, mis en ligne le 20 mars 2012, consulté le 06 octobre 2020. URL : http://journals.openedition.org/germanica/1370 ; DOI : https:// doi.org/10.4000/germanica.1370

Ce document a été généré automatiquement le 6 octobre 2020.

(c) Tous droits réservés 


\section{Bovarysme et snobisme, deux symptômes de la nervosité des nations. Étude de cas : Der Mann ohne Eigenschaften}

Till R. Kuhnle

\section{Symptômes universels d'une grande nervosité}

1 «He who meanly admires mean things is a Snob-perhaps that is a safe definition of the character" / "Celui qui admire de façon mesquine des mesquineries est un snob - peut-être est-ce là une sûre définition du genre $»^{1}$. Or cette définition donnée en 1849 par l'Anglais Thackeray dans son fameux Book of Snobs est loin de cette clarté qu'on attend d'une définition puisqu'elle est loin d'élucider ce qu'il faut entendre par « mean things » et l'adverbe « meanly " peut être compris au moins dans deux sens différents, voire contradictoires: s'agit-il d'une attitude par rapport à la chose même ou par rapport à un interlocuteur? Bien que l'étymologie du mot «snob » - à l'instar de « kitsch» - paraisse toujours incertaine, les voix qui la font remonter à «sine nobilitate» sont certainement les plus proches des significations qu'on lui attribue généralement ${ }^{2}$. Par « snobisme » on désigne donc une attitude qui cherche à se distinguer des autres sans pour autant bénéficier de la reconnaissance dite institutionnelle dont profite l'aristocratie ; cette attitude va de pair avec une ignorance relative à certains faits qui transforment en sérieux ce qui, en réalité, est le produit de la bêtise pure. Il est pourtant évident qu'on ne parle de « snob » qu'à partir de l'avènement de l'ère bourgeoise. La société bourgeoise sert donc de référence à toutes les significations éthiques ou esthétiques qu'on attribue au terme, peu importe qu'il s'agisse de décrire une manière de se distinguer au sein même de cette société ou, au contraire, une attitude qui cherche à s'en détacher complètement. Nonobstant, selon Jules Lemaître, les premiers grands snobs « reconnus » apparurent sur la scène du Grand Siècle, à savoir le Bourgeois-gentilhomme et les 
Précieuses ridicules de Molière. Ils ont pu former une espèce d'archétype puisqu'ils renvoient inexorablement à la classe qu'ils représentent. Ce qui a amené Lemaître à proposer cette définition du «snobisme » qui renoue avec Thackerey: «C'est l'alliance d'une docilité de l'esprit presque touchante et de la plus risible vanité $»^{3}$. Ainsi, le snobisme peut être associé à cette "bêtise bourgeoise " à caractère universel que dénonce Flaubert à travers ses grands romans Madame Bovary, Bouvard et Pécuchet et L'Éducation sentimentale. Notamment ce dernier connait de nombreux personnages qu'on pourrait qualifier de «snob » - bien que Flaubert ait évité ce terme.

2 En 1902, dans Le Bovarysme, Jules de Gaultier affirme l'existence d'un syndrome particulier: «le pouvoir départi à l'homme de se concevoir autre qu'il n'est. C'est [lui] que, du nom de l'une des principales héroïnes de Flaubert, on a nommé le Bovarysme $»^{4}$. Cette "pathologie du bovarysme» est le résultat d'une étude de psychologie philosophique partant de l'œuvre de Flaubert à laquelle Gaultier a déjà consacré en 1892 une première étude, Le Bovarysme. La psychologie dans l'œuvre de Flaubert, introduisant ce nouveau terme dérivé du nom Madame Bovary. Or, nourri de la lecture de Nietzsche et de Schopenhauer, mais aussi des grands moralistes d'inspiration janséniste, le philosophe français cherche à démontrer un fait anthropologique derrière ce mensonge romantique qui pousse les personnages à se choisir un modèle qu'ils imitent à la lettre. Par la suite, le terme fera son entrée dans la terminologie de la critique littéraire pour devenir une métaphore désignant la condition de l'homme comme étant celle d'un mensonge universel ${ }^{5}$.

3 C'est toutefois la situation de l'homme au tournant du xixe siècle au xxe siècle qui apparaît à travers l'œuvre de Gaultier. Il s'agit d'une situation marquée d'un profond sentiment d'aliénation: l'homme doit se rendre à l'évidence que vivre signifie désormais être interminablement en quête d'une identité, d'une place dans le monde. Or, les mêmes conditions qui le poussent à cette quête la rendent vaine et absurde puisqu'elle ne connaît aucun fondement métaphysique. Il s'ensuit que l'homme est condamné à vivre dans une bulle fondée sur une fiction qui, à son tour, repose sur le choix d'un 'modèle' à imiter afin de s'emparer d'une réalité. Mais en choisissant un tel 'modèle', l'homme se cache à lui-même que celui-ci dépend uniquement de son imagination et ne désigne donc aucune vérité existant en dehors de cette imagination.

4 En 1911, le néo-kantien Hans Vaihinger publie son œuvre fondamentale Die Philosophie des Als-Ob / La Philosophie du «comme si » qui souligne que la conscience est double, car elle est à la fois la source des fictions et l'instance censée porter un jugement sur leur validité ; or, comme toute connaissance trouve son origine dans la fiction, on peut donc conclure avec Vaihiger, qui se situe bien dans les sillages à la fois de Kant et de Nietzsche, que l'homme ne saura jamais saisir sa véritable identité. L'évidence de la séparation entre sujet et objet est alors mise en cause à un tel point que l'immédiat du vécu est posé comme unique réalité certaine ${ }^{6}$. L'aliénation de l'individu est donc scellée. Nul ne représente mieux cette conditio moderna que le nouvel anti-héros romanesque. Depuis le romantisme, selon Lukács, "la forme romanesque traduit plus que toute autre ce qui est transcendentalement ni feu ni lieu » / [...] « die Form des Romans ist wie keine andere Ausdruck der transzendentalen Obdachlosigkeit $»^{7}$.

5 Vaihinger se rapproche d'ailleurs de Jules de Gaultier qui a parlé d'une "fiction universelle » à l'intérieur de laquelle agissait un étrange pouvoir : «Le pouvoir de la 
fiction bovaryque pour attirer l'homme vers elle se fonde, semble-t-il, sur cet amour de soi où La Rochefoucauld a vu le principe fondamental de toute activité humaine $»^{8}-$ en d'autres termes, l'homme fait en sorte que «le réel sort[e] de l'irréel » afin de défendre les intérêts individuels ${ }^{9}$. Fiction et mensonge font donc partie d'une même stratégie dans la lutte pour la conservation.

6 Le livre de Vaihinger est sorti à peine une douzaine d'années après une autre œuvre qui avait commencé à ébranler ce siècle naissant: Die Traumdeutung / L'interprétation des rêves (1899/1900) de Sigmund Freud dont les travaux ont appris à l'homme " que le moi n'est même plus maître chez soi » / "daß das ich nicht einmal mehr Herr in seinem eigenen Haus ist $»^{10}$. Et la théorie des civilisations développée par Freud démontre que celles-ci sont fondées sur la sublimation (une image empruntée à Nietzsche ${ }^{11}$ ), sur un principe qui diffère la satisfaction des pulsions en échangeant leur objet $^{12}$. Ainsi, le mensonge est parallèle au processus de la civilisation, il est à l'origine de ce «malaise de la civilisation» dont fut empli Freud ayant dénoncé « l'avenir d'une illusion $»^{13}$.

7 Ce xxe siècle qui, lors de la parution des études importantes de Gaultier, de Freud ou de Vaihinger, vient de commencer est donc marqué par une maladie héritée du siècle précédent, par une culture des masses qui renie l'individu. L'ennui, ce grand mal du xIxe siècle, a produit dans les pays européens différentes formes d'apparences extérieures et des excitations affectives qui traduisent le désir de s'élever au-dessus de la foule, de s'imposer à la manière de cette noblesse qui a perdu son aura et d'opposer à un monde du travail frappé par l'aliénation un style de vie distingué ${ }^{14}$. En 1909, Carl Einstein considère le snob comme le produit d'un monde en crise de valeurs, comme l'expression nerveuse du vouloir individuel («überreiztes Individualwollen»), une attitude veule et dépourvue de la puissance de la Volonté - au sens nietzschéen du terme ${ }^{15}$. Le snobisme sera désormais au nombre des éléments caractéristiques d'une fin de siècle marquée par un arrivisme froid ${ }^{16}$, mais aussi par le goût du luxe dont l'art nouveau est une des nombreuses formes d'expression. Une vingtaine d'années après Carl Einstein, Walter Benjamin ira encore plus loin en dénonçant le snob proustien comme l'apogée de l'esprit consommateur ${ }^{17}$ tourné vers la nouveauté dont il devient l'arbitre - « arbiter novarum rerum $»^{18}$.

Or, les différentes approches du phénomène du snobisme esquissées ici - et la liste est loin d'être exhaustive - suggèrent en grande partie des analogies entre des symptômes pathologiques et des phénomènes culturels. Ainsi, le snobisme et le bovarysme, dont il sera encore question plus tard, comptent parmi les symptômes les plus importants d'une «névrose" qui paraît avoir frappé définitivement toute la bourgeoisie européenne depuis la fin du xixe siècle.

9 Il est étonnant que Gaultier ne soit pas cité dans la plupart des études portant sur le snobisme, d'autant plus que son livre de 1902 contient un chapitre qui, malgré sa brièveté, précise les caractères essentiels de cette attitude devenue un phénomène universel au sein même d'une société à la recherche d'une défense et illustration de l'individu face à une culture des masses naissante. Gaultier y établit une distinction qui, à première vue, paraît étonnante : «L'homme de génie se conçoit autre qu'il n'est par excès d'énergie, le snob fait de même par défaut. C'est un débile et il sait ce qu'est la force $»^{19}$. Le snob apparaît donc comme quelqu'un qui évite le risque afin de préserver les apparences. C'est sa stratégie de survie. Contrairement au génie, «il évite les œuvres ». Autrement dit, le snob adopte l'attitude du dilettante: «Refugié dans les 
admirations, il tire sa gloire personnelle des œuvres des autres $»^{20}$. Mais ce dilettante cherche pourtant à se distinguer et "lorsqu'il s'attache soit à quelque opinion, soit à quelque nom de philosophe, d'écrivain ou d'artiste, c'est la nouveauté et la rareté du choix, c'est l'obscurité de l'objet sur lequel il se fixe ${ }^{21}$ qui prime. Il cherche donc à compenser son manque d'énergie et de vitalité par la seule apparence censée le justifier. Ou pour le dire avec Nietzsche : il se range du côté d'un faux apollonien. La perfection de la mise en scène amène Gaultier à conclure que le snobisme «est un Bovarysme triomphant, c'est l'ensemble des moyens employés par un être pour s'opposer à l'apparition, dans le champ de la conscience, de son être véritable, pour y faire figurer sans cesse un personnage plus beau en lequel il se reconnait ${ }^{22}$. On est tenté d'y voir le résultat d'une névrose au sens psychanalytique du terme - à savoir d'une «Affection psychogène résultant d'un conflit inconscient entre les désirs du sujet et les interdits qui s'opposent à leurs réalisations ${ }^{23}$. Mais Nietzsche associe la névrose au génie, afin de la distinguer de l'hystérie qu'il a cru découvrir chez les artistes efféminés de son temps ${ }^{24}$. Ceci montre, à quel point une nosographie des phénomènes culturels dépend du point de vue de celui qui l'applique - les jugements portés sur le personnage de Madame Bovary pourraient en témoigner.

10 L'incontournable 'vérité' des mensonges relevés par les auteurs cités a fait son apparition au moment de l'écroulement d'un univers - comme celui de l'Empire austrohongrois à la suite de la Grande Guerre. C'est à cette époque qu'Alfred Adler, renouant avec son étude fondamentale sur « le tempérant nerveux » de 1912 (Über den nervösen Charakter / Le tempérament nerveux), publie deux livres, Menschenkenntnis / Connaissance de l'homme (1927) et surtout Der Sinn des Lebens / Le sens de la vie (1933), qui rendront populaires quelques concepts de sa psychologie individuelle («Individualpsychologie »), notamment le « complexe d'infériorité » et le « complexe de supériorité ». Ce dernier "apparaît le plus souvent nettement dessiné dans l'attitude, les traits de caractère et l'opinion de l'individu, persuadé de ses propres dons et capacités, supérieurs à la moyenne de l'humanité ${ }^{25}$. Dans la longue liste des symptômes - nourris avant tout d'un sentiment d'infériorité - qu'on peut trouver chez Adler apparait aussi le « snobisme $»^{26}$.

11 S'appuyant sur la psychologie individuelle, Werfel constate vers la fin des années 20 le règne d'une grande puissance nouvelle:Der Snobismus als geistige Weltmacht. Selon Werfel, le snobisme est une maladie psychique difficile à définir, mais dont les germes se trouvent en chaque individu ${ }^{27}$. Afin d'en expliquer le mécanisme, il a recours à l'idée nietzschéenne d'une volonté de puissance. Vivre cette volonté implique tout d'abord la volonté de prendre le pouvoir ("der Wille zur Machtergreifung ») et donc d'en assumer les impératifs. Or, l'individu qui rêve du pouvoir ne rêve que de l'image du pouvoir, de la pompe et de la gloire qu'il promet. Bref, il ne cherche que la reconnaissance ("Wille zur Geltung »), le besoin de se faire valoir prime toute autre activité. Ainsi le snobisme n'est rien d'autre que «le besoin de se faire valoir devenu pathologique» / «der krank gewordene Trieb zu Geltung $»^{28}$. Par la suite, Werfel énumère les nombreux symptômes de cette "maladie» comme le culte de la nouveauté, le choix des choses exquises ou d'un style qui lui épargne la nécessité de se faire comprendre, l'excentricité, la perversité, le culte du crime, etc. Mais il cerne aussi le snobisme dans les positions politiques extrêmes : le révolutionnaire apparaît comme le snob par excellence car il totalise les normes d'une société sous l'emprise du matérialisme, tout en faisant valoir une position de pure négativité. Le besoin de se faire valoir devenu pathologique tend de gré ou de force vers des idéaux malades, il est 
le symptôme même de la crise : «Er [der Geltungswahn] ist das Ekzem, der Ausschlag, das Symptom, welches die Krise anzeigt ${ }^{29}$. L'essai de Franz Werfel permet donc une première conclusion : le snobisme, cette "geistige Weltmacht ", est l'expression d'une crise profonde des sociétés européennes. Selon Habermas, on parle de crise « lorsque la structure d'un système social affronté à un problème admet moins de possibilités de solutions que le système n'en réclame pour se maintenir $"{ }^{30}$. Une telle situation de crise est le point de départ de l'étude de cas qui suit.

\section{Étude de cas : Der Mann ohne Eigenschaften}

En août 1913, dans la métropole de l'Empire des Habsbourg, le jeune "intellectuel » viennois Ulrich décide de "prendre congé de la vie». Son père, peu convaincu de son fils, l'encourage à participer aux préparatifs du 70e anniversaire de l'intronisation de l'empereur autrichien François-Joseph qui est censé être commémorée par une grande cérémonie chargée de symbolisme, d'autant plus que ce sera le 30e anniversaire de l'intronisation de l'empereur allemand Guillaume II. Ainsi, cette double commémoration qui aura lieu en 1918 est préparée de concert: c'est une «Parallelaktion» (« action parallèle »). Le jeune homme s'impose tant bien que mal au sein des «salons" dont les membres sont pourtant loin d'être à la hauteur d'un tel projet. La «Parallelaktion» n'est qu'un prétexte, une fiction nécessaire à la constitution d'un groupe ; et le lecteur sait d'ores et déjà - sans connaître la fin du roman resté fragment - que l'Histoire enlèvera au groupe la possibilité de réaliser ce projet. L'inertie mêlée à ses nombreux talents fait du jeune désœuvré de bonne famille cet Homme sans qualités - Mann ohne Eigenschaften ${ }^{31}$ - qui, en effet, est un homme empêché par une "mystérieuse maladie de l'époque » / " eine geheimnisvolle Zeitkrankheit » de faire valoir ses nombreuses qualités :

Ainsi donc, l'époque avait changé, comme un jour qui commence radieux et insensiblement se couvre, et elle n'avait pas même eu la politesse d'attendre Ulrich. Il le lui rendait bien en expliquant les mystérieux changements qui constituaient sa maladie et consumaient son génie, par la plus ordinaire des bêtises. Mais cela sans aucune intention blessante. Si la bêtise, en effet, vue du dedans ne ressemblait pas à s'y méprendre au talent, si, vue du dehors, elle n'avait pas toutes les apparences du progrès, du génie, de l'espoir et de l'amélioration, personne ne voudrait être bête et il n'y aurait pas de bêtise. Tout au moins serait-il aisé de la combattre. Le malheur est qu'elle ait quelque chose d'extraordinairement naturel et convaincant. (Homme, 73sq).

Le ton de la description est décidément crépusculaire. Et ses réflexions sur la bêtise, qui évoquent bien la bêtise bourgeoise dénoncée par Flaubert et plus encore le snobisme dans l'acceptation la plus large du terme, culminent dans l'affirmation d'une sorte de théodicée qui parait justifier la bêtise en tant que catalyseur de la vérité. Or, c'est le contraire qui se produit : étant imitée à la lettre, c'est notamment la bêtise qui pousse le mensonge universel jusqu'à la catastrophe.

Dans L'Homme sans qualités, où les protagonistes agissent dans un climat de catastrophe imminente, l'essai prime la narration - et c'est dans le même roman que Musil développe sa notion d'Essayismus / essayisme en définissant l'essai non seulement comme un genre littéraire mais comme une attitude :

[...] un essai est la forme unique et inaltérable qu'une pensée peu décisive fait prendre à la vie intérieure d'un homme. Rien n'est plus étranger à l'essai que l'irresponsabilité et l'inachèvement des inspirations qui relèvent de la subjectivité ; 
pourtant les notions de vérité et d'erreur, d'intelligence ou de sottise ne sont pas applicables à ces pensées soumises à des lois non moins strictes qu'apparemment subtiles et ineffables. Assez nombreux furent ces essayistes-là, ces maîtres du flottement intérieur de la vie; il n'y aurait aucun intérêt à les nommer; leur domaine se situe entre la religion et le savoir, entre l'exemple et la doctrine, entre l'amor intellectualis et le poème ; ce sont des saints avec ou sans religion et parfois, aussi simplement des hommes égarés dans telle ou telle aventure (Homme, 320) ${ }^{32}$.

Le ton de ce passage est bien ironique ! Et avant de continuer, il faut distinguer l'ironie du mensonge : l'ironie présuppose que la vérité est connue - l'ironie est une stratégie rhétorique pour faire valoir la vérité au prix de porter atteinte à la vraisemblance d'un énoncé. Le mensonge et la ruse, par contre, opèrent sur la dissimulation de la vérité pour atteindre un but; une fois dévoilé comme mensonge, un énoncé perd définitivement toute plausibilité ou vraisemblance. Dans leur finalité, l'ironie et le mensonge se rejoignent : à la rigueur, on peut atteindre le même but avec l'ironie ou le mensonge. Au moins en rhétorique, l'union intime des deux principes est bien connue d'autant plus quand les paramètres de la vérité commencent à devenir incertains : l'ironie et le mensonge se rapprochent de plus en plus puisque la distinction entre les deux présuppose une distinction nette entre vérité et mensonge ${ }^{33}$.

Or, ne proposant que des possibilités, le discours essayiste détruit toute notion de totalité afin d'accéder au refus de figer son objet dans un concept (all. Begriff) ${ }^{34}$. L'« utopie de l'essayisme » posée comme absolue ne vaut donc pas plus que l'utopie « de la vie réelle ", à savoir la totalisation du monde existant ! Dans le roman, le personnage est toujours doublé par les instances narratives qui transforment les éléments du texte en essai au moment où ils s'éloignent de leur personnage - Adorno a souligné qu'un mauvais essai parlait de personnes au lieu de saisir la chose ${ }^{35}$. L'essai ne propose donc pas une vérité, mais il dénonce le mensonge, même celui qui se cache derrière un simple bilan de comptable.

Ce qui est mis en œuvre ici, c'est ce qu'on peut appeler le paradoxe de la déclaration d'impôts. Après avoir établi une déclaration d'impôts, on reçoit un avis de la trésorerie, une somme arrondie à deux chiffres derrière la virgule. Or, cette précision repose pourtant sur une pure fiction: elle implique qu'on réussisse à indiquer avec la même précision tous les revenus et toutes les dépenses. Autrement dit: la déclaration d'impôts a une valeur purement rhétorique ! Mais avec des effets bien réels...

Une telle précision doit être considérée, selon Musil, comme une attitude humaine qui " pose à l'être et au faire des exigences maxima ». Mais ceci demande à être nuancé :

En effet, il n'existe pas seulement une précision imaginaire (qui d'ailleurs, dans la réalité, n'existe encore nullement), mais une précision pédante, et ces deux espèces de précisions se distinguent en ceci que l'imaginaire s'en tient aux faits et la pédante à des créations de l'imagination (Homme, 312) ${ }^{36}$.

19 Il va de soi que nulle précision ne saura mener à la vérité. Tant que l'homme ne s'avoue pas à lui-même cet échec, toute quête de vérité n'est pas à la hauteur de cette utopie nommée "l'utopie de l'essayisme » qui ne connaît qu'un seul impératif éthique : «Pas plus qu'on ne peut faire des parties authentiques d'un essai une seule vérité, on ne peut tirer d'un tel état une conviction ; du moins pas sans devoir aussitôt l'abandonner [...] » $\left(\right.$ Homme, 322) ${ }^{37}$. En effet, il va de soi que L'Homme sans qualités rendant hommage à cette utopie ne saura jamais la vivre car il est pris dans le mensonge qui s'installe tant que les subjectivités n'arrivent pas à communiquer sans médiation. 
Ulrich n'est pas près de vivre l'essayisme, ou au contraire : par le fait même d'imposer l'essayisme comme sa vérité, il trahit celui-ci en faveur de son snobisme.

En vérité, le snob est bien loin du génie qui s'exprime à travers une œuvre. Par ailleurs, en tant qu'homme sans qualités, Ulrich n'appartient pas à cette avant-garde qui, selon Adorno, est nourrie du «'snobisme' de la connaissance la plus avancée ", c'est-à-dire d'un esprit critique dénoncé comme «snob " par les réactionnaires. Ce 'snobisme', qui est celui d'un Picasso, d'un Stravinski ou d'un Proust, apparaîtrait un jour, dans un autre contexte, sous un aspect bien différent par rapport à l'ordre bourgeois, à savoir «comme l'anticipation dialectique de la disparition de celui-ci » / "als dialektische Vorwegnahme von deren Untergang ${ }^{38}$. Cette inversion dialectique du snobisme marque aussi sa lecture de Proust qui l'amène au constat que le snobisme et l'art nouveau ont ceci en commun : ils sont deux tentatives de transcender les conditions de la classe bourgeoise qui se définit par rapport aux lois du marché afin de se projeter dans l'image d'une beauté échappant à ses lois ${ }^{39}$. Par ailleurs, Adorno renoue avec les catégories freudiennes afin de caractériser le snobisme, à savoir le snobisme de Proust, comme une forme d'érotisation de faits sociaux - "die erotische Besetzung gesellschaftlicher Tatbestände » - qui cherche la provocation à l'encontre d'une vie tournée vers le faux ${ }^{40}$.

21 Ce n'était donc pas pour rien que Jules Lemaître se demanda en 1899 si son article sur le snobisme littéraire serait "pour en faire la satire ou l'apologie » ${ }^{41}$. Les écrits sur le snobisme - de Thackeray à Adorno - ont ceci en commun qu'ils dénoncent la faiblesse de l'individu atteint d'un sentiment de manque, d'une grande nervosité ou d'une névrose. Les termes de la nosologie psychiatrique peuvent varier, voire développer des sens contradictoires, mais lorsqu'ils sont appliqués au snobisme le constat reste le même : il s'agit d'une expression pathologique du monde bourgeois derrière laquelle se cache un choix existentiel de l'individu, «se concevoir autre qu'il n'est» (J. de Gaultier). Or, il s'agit d'un " pouvoir » qui paraît connaître deux issues, comme l'a déjà souligné Nietzsche, à savoir la névrose ou l'hystérie, idée reprise par Jules de Gaultier qui distingue par «l'excès d'énergie » le génie du snob. Mutatis mutandis, cette approche trouvera également son écho chez Franz Werfel et, d'une manière moins évidente, chez Adorno dont la dialectique négative doit beaucoup à la critique nietzschéenne de l'humain, trop humain ${ }^{42}$. Même dans les écrits de Nietzsche apparaissent les apories concernant le rôle de la névrose dans les différentes théories de la civilisation qui, malgré leurs prétentions et les discursivités qu'elles fondent, s'avèrent incapables de figer leur objet dans un concept (Begriff). Seul l'essayisme peut donc se saisir d'une manière adéquate du snobisme - dont il est, au moins dans l'univers de Der Mann ohne Eigenschaften, à la fois symptôme et remède.

L'homme - à savoir le bourgeois - est donc condamné à vivre dans un monde de mensonges; et il périra avec lui. Ulrich, l'homme sans qualités, vit dans une période de grande nervosité, dans un univers condamné à disparaître. Un tel univers ne connaît qu'un seul espoir, celui de l'avènement d'un monde meilleur après le cataclysme. « $\mathrm{Ce}$ fut donc une période réellement messianique que celle qui précéda la Grande Guerre » écrit Musil. En effet, le lecteur de Der Mann ohne Eigenschaften sait que les personnages agissent sous le signe de l'Apocalypse imminente, de ce cataclysme qui mettra fin à l'insoutenable légèreté d'un monde qui enlève à toute activité humaine sa raison d'être. Mais les rêves de rédemption restant rattachés aux visions particulières des individus ou des groupes ne sauront jamais être que des rêves qui évoquent les 
apparences d'une rédemption sans pour autant saisir la Rédemption. Il s'ensuit que la Première Guerre mondiale a une fois pour toutes scellé le règne du snobisme comme cette " geistige Weltmacht » dénoncée par Werfel. C'est ainsi qu'il faut comprendre ces lignes de Musil :

Ainsi s'étaient-ils finalement persuadés que l'époque dans laquelle ils vivaient était vouée à la stérilité intellectuelle, et ne pouvait en être sauvée que par un événement ou un homme tout à fait exceptionnels. C'est alors que naquit, parmi ceux qu'on appelle les intellectuels, le goût du mot " rédimer ». On était persuadé que la vie s'arrêterait si un messie n'arrivait pas bientôt. C'était, selon le cas, un messie de la médecine [...] ou un messie de la poésie qui devait être en mesure d'écrire un drame qui attirerait des millions d'hommes dans les théâtres et serait cependant parfaitement original dans sa noblesse spirituelle. En dehors de cette conviction qu'il n'était pas une seule activité humaine qui pût être sauvée sans l'intervention d'un messie particulier, existait encore, bien entendu, le rêve banal et absolument brut d'un messie à la manière forte pour rédimer le tout (Homme, $655)^{43}$.

Autrement dit: l'espèce humaine est celle qui sait mentir! En principe, elle peut prendre sa rédemption en main. Mais il reste cet étrange phénomène pathologique constaté par Jules de Gaultier : «Le Bovarysme est la faculté départie à l'homme de se concevoir autre qu'il n'est en tant que l'homme est impuissant à réaliser cette conception différente qu'il se forme de lui-même $\star^{44}$.

\section{NOTES}

1. W[illiam]. M[akepeace]. Thackeray: Le Livre des Snobs / The Book of Snobs [édition bilingue], traduit et préfacé par Raymond Las Vergnas, Paris : Aubier / Montaigne 1955, 68sqq.

2. Philippe du Puy de Clinchamps donne une première introduction à l'histoire du snobisme : Le Snobisme, Paris : PUF (Que sais-je ?) 1964 ; pour les références en langue allemande, c'est avant tout l'entrée « Snob. Snobisme » d'A. Kosenina dans Joachim Ritter (dir.) : Historisches Wörterbuch der Philosophie, Darmstadt : wbg 1971-2007. Pour le contexte français sont à citer les ouvrages suivants : Émilien Carassus : Le Snobisme et les lettres françaises de Paul Bourget à Marcel Proust. 1884-1914, Paris : Armand Colin 1966 ; Frédéric Rouvillois : Histoire du snobisme, Paris : Flammarion (Champs histoire) 2010.

3. Jules Lemaître : «Les Snobs ", in : Les Contemporains. Études et portraits littéraires. $7^{\mathrm{e}}$ série, Paris : Société française d'imprimerie et de librairie (ancienne librairie Eugène Oudin) 1899, 96.

4. Jules de Gaultier: Le Bovarysme, suivi d'une étude de Per Buvik, "Le Principe bovaryque ", Paris : PUPS 2006, 10.

5. Cf. l'état de recherche de Pierre Buvik: «Le principe bovaryque », in : Jules de Gaultier : op. cit. Il faut noter avant tout l'étude de René Girard: Mensonge romantique et vérité romanesque, Paris : Hachette (pluriel) 1999 [1 $1^{\text {ère }}$ éd. 1961].

6. Hans Vaihinger : Die Philosophie des Als ob. System der theoretischen, praktischen und religiösen Fiktion der Menschheit auf Grund eines idealistischen Positivismus, Leipzig : $8^{\mathrm{e}}$ éd., 1922, 287 (pour la version française cf. La Philosophie du "Comme si », préface et traduction de Christophe Bauriou, Paris : Kimé 2008) ; cf. aussi le chapitre « Fiktion und inauthentische Setzung (Hans Vaihinger)», in : Wolfgang Iser : Das Fiktive und das Imaginäre. Perspektiven einer literarischen Anthropologie, 
Frankfurt a. M. : Suhrkamp 1991, 231. L'« immédiat du vécu » est aussi le point de départ de la Lebensphilosophie (philosophie de la vie) - à savoir celle d'Henri Bergson - précédant la philosophie de l'existence.

7. Georg Lukács: La Théorie du roman, traduit par Jean Clairevoye, Paris: Denoël / Gallimard (coll. Tel) 1989, 32. / Die Theorie des Romans. Ein geschichtsphilosophischer Versuch über die Formen der großen Epik, Darmstadt / Neuwied : Luchterhand (Sammlung Luchterhand) $9^{e}$ éd., 1984, 32.

8. J. de Gaultier : La Fiction universelle. Deuxième essai sur le pouvoir d'imaginer, Paris : Mercure de France 1903, 24.

9. Id., 26.

10. Sigmund Freud: Vorlesungen zur Einführung in die Psychoanalyse ( = Gesammelte Werke XI), éd. par Anna Freud, Frankfurt a. M. : Fischer (réimpression en poche) 1999, 295.

11. Friedrich Nietzsche : Menschliches, Allzumenschliches I und II ( = KSA 2), éd. par Giorgio Colli und Mazzino Montinari, München : dtv 1988, 414sq.

12. Sigmund Freud : "Die 'kulturelle' Sexualmoral und die moderne Nervosität », in : Gesammelte Werke VII, op. cit., 141-167, 150.

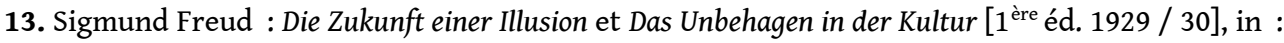
Gesammelte Werke XIVI, op. cit., 323-380 et 419-406.

14. Cf. l'anthologie établie par Gerd Stein : Dandy, Snob, Flaneur, Dekadenz und Exzentrik. Kulturfiguren und Sozialcharaktere des 19. und 20. Jahrhunderts: Frankfurt a. M., Fischer Taschenbuch Verl., 1985.

15. Carl Einstein : «Der Snob[b] », in : Werke I, texte établi par Rolf-Dieter Baake, Berlin : Medusa 1980, 23-27, 24.

16. Cf. la pièce Der Snob de Carl Sternheim, sortie en février 1914.

17. Walter Benjamin : "Zum Bilde Prousts [1929]», in : Gesammelte Schriften II, texte établi par Rolf Tiedemann und Herbert Schweppenhäuser, Frankfurt a. M. : Suhrkamp (stw) 1991, 310-324, 319.

18. W. Benjamin : "Paris, die Hauptstadt des 19. Jahrhunderts", in: Gesammelte Schriften V (= Passagen-Werk), op.cit., 45-59, 56. Le snob figure ici avant tout comme instance du jugement esthétique, par opposition au dandy : « Er [der Snob] ist der Kunst, was der Dandy der Mode ist » (id.). La version française (id. 60-77) de ce texte ne contient pas les remarques sur le snob!

19. J. de Gaultier : Le Bovarysme, op. cit., 45. Cf. dans ce contexte aussi le texte satirique de W. Benjamin : «Was schenke ich einem Snob », in : Gesammelte Schriften III, op. cit., 209-211.

20. Id., 46.

21. Id., 48.

22. Id., 49.

23. Art. « Névrose ", in : Trésor de la langue française (TLF).

24. F. Nietzsche : Nachgelassene Fragmente. 1887-1889 ( = KSA 13), op. cit., § 14 [119] : « Wie man heute "Genie" als eine Form der Neurose beurtheilen dürfte, so vielleicht auch die künstlerische Suggestions-Kraft, - und unsere Artisten sind in der That den hysterischen Weiblein nur zu verwandt!».

25. Alfred Adler : Le Sens de la vie. Étude de psychologie individuelle comparée, trad. par Herbert Schlaffer, Paris : Payot (petite bibliothèque) 1968, 90.

26. Id., 91.

27. Franz Werfel : Der Snobismus als geistige Weltmacht, in : Zwischen oben und unten. Prosa, Tagebücher, Aphorismen, literarische Nachträge, München / Wien : Langen-Müller 1975, 261-278, 262.

28. Id., 265.

29. Id., 277. 
30. Jürgen Habermas : Legitimationsprobleme im Spätkapitalismus, Frankfurt a. M. : Suhrkamp (éd.) 1973, 11 ; version française : Raison et légitimité. Problèmes de légitimation dans le capitalisme avancé, traduit par J. Lacoste, Paris : Payot 1978, 13.

31. Robert Musil : L'Homme sans qualités (2 vol.), traduit par Philippe Jaccottet, Paris, Seuil coll. « Points » 1995 - cité Homme dans le texte. Robert Musil : Der Mann ohne Eigenschaften (2 vol. ), éd. par Adolf Frisé, Reinbek (Hamburg), Rowohlt (Sonderausgabe) 1981 - cité Mann dans les notes.

32. «[...] ein Essay ist die einmalige und unabänderliche Gestalt, die das innere Leben eines Menschen in einem entscheidenden Gedanken annimmt. Nichts ist dem fremder als die Unverantwortlichkeit und Halbfertigkeit der Einfälle, die man Subjektivität nennt aber auch wahr oder falsch, klug und unklug sind keine Begriffe, die sich auf solche Gedanken anwenden lassen, die dennoch Gesetzen unterstehen, die nicht weniger streng sind, als sie zart und unaussprechlich erscheinen. Es hat nicht wenige solcher Essayisten und Meister des innerlich schwebenden Lebens gegeben, aber es würde keinen Zweck haben, sie zu nennen ; ihr Reich liegt zwischen Religion und Wissen, zwischen Beispiel und Lehre, zwischen amor intellectualis und Gedicht, sie sind Heilige mit und ohne Religion und manchmal sind sie auch einfach Männer, die sich in ein Abenteuer verirrt haben » (Mann, 253sq.)

33. On reproduit ici la distinction entre ironie et mensonge établie par Jean-Pol Madou : "Ironie socratique, ironie romanesque, ironie poétique », in : Philippe Desan et al. (dir.) : Irony and Satyre in French Literature, Columbia : The University of South Carolina 1987, 62-73, 62sq.

34. Cf. Peter V. Zima: Roman und Ideologie. Zur Sozialgeschichte des modernen Romans, München: Fink 1987, 67, et Georg(es) [György] Lukács : Die Seele und die Formen, Berlin : Egon Fleischel 1911 / L'Âme et les formes, traduit de l'allemand par Guy Haarscher ; notes introductives et postface par Guy Haarscher, Paris : Gallimard 1974.Un important texte de référence qui reprend les considérations de Lukács caractérisant l'essai comme la recherche d'une forme de représentation de ce qui est déjà connu et non pas comme une source de connaissance (Seele und Form, 1911) est « Der Essay als Form » qui ouvre les Noten zur Literatur ( = Gesammelte Schriften 11, Frankfurt a. M. : Suhrkamp 1977, 11-22) de Theodor W. Adorno ; cf. aussi l'état de recherche de Klaus Beekman: «Essay Essayismus und die Grenzen der modernen Literatur», in: Avant-Garde $n^{\circ} 0$, Revue interdisciplinaire et internationale. Arts et littératures au $\mathrm{xx}^{\mathrm{e}}$ siècle. Interdisciplinary and International Review of Literature and Arts oft 20th Century, Amsterdam : Rodopi 1988, 15-26.

35. Th. W. Adorno, "Der Essay als Form ", op. cit., 13.

36. «Denn in Wirklichkeit gibt es ja nicht nur die phantastische Genauigkeit (die es in Wirklichkeit noch gar nicht gibt), sondern auch eine pedantische, und diese beiden unterscheiden sich dadurch, daß sich die phantastische an die Tatsachen hält und die pedantische am Phantasiegebilde. » (Mann, 247)

37. «Und so wenig man aus den echten Teilen eines Essays eine Wahrheit machen kann, vermag man aus einem solchen Zustand eine Überzeugung zu gewinnen ; wenigstens nicht, ohne ihn aufzugeben. » (Mann, 255).

38. Th. W. Adorno : «Gegen die neue Tonalität», in: Musikalische Schriften V (= Gesammelte Schriften 18), op. cit., 101.

39. Th. W. Adorno : «Requiem für Odette » in : Minima Moralia. Reflexionen aus dem beschädigten Leben ( = Gesammelte Schriften 4), op. cit., 216: "Daher die Verwandtschaft von Snobismus und Jugendstil, dem Versuch der durch den Tausch definierten Klasse, sich ins Bild einer vom Tausch reinen, gleichsam vegetabilischen Schönheit zu projizieren». C'est ainsi qu'il peut écrire à propos de Proust que la régression de Proust porte la marque de l'utopie : «Prousts Regression ist ein Stück Utopie » (« Kleine Proust-Kommentare », in : Gesammelte Schriften 11, op. cit., 211).

40. Th. W. Adorno : « Kleine Proust-Kommentare », in : Gesammelte Schriften 11, op. cit., 211.

41. J. Lemaître : «Les snobs », op. cit., 96.

42. Cf. T. R. Kuhnle: "„Es gibt kein richtiges Leben im falschen“. Ein Versuch zu Adorno, Nietzsche und Port Royal», in: Hanspeter Plocher / Till R. Kuhnle / Bernadette Malinowski 
(dir.) : Esprit civique und Engagement. Festschrift für Henning Krauss zum 60. Geburtstag, Tübingen : Stauffenburg 2003, 358-383.

43. "Auf diese Weise entstand damals unter den sogenannten intellektuellen Menschen die Beliebtheit der Wortgruppe Erlösung. Man war überzeugt, daß es nicht mehr weitergehe, wenn nicht bald der Messias komme. Das war ja nachdem ein Messias der Medizin [...]; oder ein Messias der Dichtung, der imstande sein sollte, ein Drama zu schreiben, das Millionen Menschen in die Theater reißen und dabei voraussetzungslosester geistiger Hoheit sein sollte : und außer dieser Überzeugung, daß eigentlich jede einzelne menschliche Tätigkeit nur durch einen besonderen Messias sich selbst wieder zurückgegeben werden könne, gab es natürlich auch noch das einfache und in jeder Weise unzerfaserte Verlangen nach einem Messias der starken Hand. » (Mann, 519sq.)

44. J. de Gaultier : Le Bovarysme, op. cit., 108.

INDEX

Mots-clés : bovarysme

oeuvrecitee Der Mann ohne Eigenschaften

\section{AUTEURS}

\section{TILL R. KUHNLE}

Université de Limoges 